\title{
Feeding Practices among Infants in a Rural Community in Bangladesh: A Cross-Sectional Study
}

Rajat Das Gupta.

\begin{abstract}
Background: Proper feeding practices during infancy are necessary for the growth and development of infants and to prevent malnutrition. This study was conducted to describe the feeding practice among infants in a rural area in Bangladesh. Methods: A cross-sectional study was conducted between February and June 2013. Data was collected through face-to-face interviews of 212 mothers using a pretested questionnaire. Results: Exclusive breast feeding and complementary feeding rates were $40.6 \%$ and $97.3 \%$, respectively. One third of the mothers practiced prelactal feeding, and honey was the most common item. Maternal illness (72.7\%) was the most common reason for not giving breast milk. Infant formula was used as an alternative food in majority of the cases (72.7\%). Conclusion: Percentage of exclusive breast feeding was not satisfactory. Encouragement of female education is recommended to improve feeding practices and infant care.
\end{abstract}

Keywords: Breast Feeding, Infant, Feeding Behaviour, Bangladesh, Hand Disinfection (Source: MeSH-NLM).

\section{Introduction}

Infancy is defined by pediatricians as the time from birth until one year of age. The word itself is of Latin origin and means "unable to speak". This period is very critical, as it represents the earliest stage of rapid physical growth and acquisition of gross and fine motor skills (including sitting without assistance, walking while holding on to furniture and using pincer grasp) and cognitive functions (including imitating gestures and using familiar objects properly)., ${ }^{1,2}$

Proper feeding practices during infancy are essential for initiating and maintaining good health, nutrition and development of infants. ${ }^{3}$ According to the latest World Health Organization (WHO) statistics, $45 \%$ of deaths during infancy are due to under-nutrition (Available from: http://www.who.int/mediacentre/ factsheets/fs342/en/, updated 2014 Feb; cited 2014 Oct 10). Das Gupta is an intern doctor working at Dhaka Medical College Hospital, Dhaka, Bangladesh. He completed his MBBS at Dhaka Medical College in May 2014
Submission: May 5, 2014

Acceptance: Sep 15, 2014

Process: Peer-reviewed
Morbidity and mortality secondary to poor nutrition can be prevented through good feeding practices during the first two years of human's life. ${ }^{4}$ Breast feeding provides infants with ideal nourishment, stimulates their immune system and protects them from various infections and diseases. Breast feeding also improves response to vaccinations and provides many health-enhancing molecules, enzymes, proteins, and hormones, which are present in both the colostrum and breast milk. ${ }^{5}$ Colostrum is the milk produced in late pregnancy and the first 2-3 days postpartum.

Unfortunately, in the majority of cases, exclusive breast feeding is not practiced in Bangladesh. Most of the neonates in Bangladesh receive prelacteal feeding, which is food given to neonates before the initiation of lactation. Prelacteal feeding includes sugar water, honey, or milk other than breast milk. ${ }^{3}$
Many studies have been conducted to evaluate the feeding practices among infants in Bangladesh. However, there have been no studies conducted that show feeding practice patterns. This study was conducted to describe the feeding practices among infants in a rural community of Bangladesh. The results may be used to recommend the necessary steps to be taken by the authorities to improve early nutrition in infants.

\section{Methods}

A cross-sectional study was conducted between February 2013 and June 2013 in Copalpur union under Begumganj Upazila (sub district) of Noakhali district in Bangladesh. The initial population included any mothers with infants. Mothers willing to take part in the study were included after giving informed consent. Mothers who were not willing to take part in the study were excluded. Given that the national rate of exclusive breast feeding in Bangladesh is quoted at $25 \%$ and considering a confidence interval of $95 \%$, the sample size should be $288 .{ }^{6}$ of the 710 households in the Gopalpur Village, 300 households were sampled using systematic random sampling. Only 212 mothers were willing to take part in the study and that was the final sample size.

Data were collected through face-to-face interviews of the respondents with the help of a pretested semi-structured interview schedule. There were two parts: questions relating to socio-demographic characteristics and questions related to feeding practices. The questionnaire was validated (unpublish data) and a biostatistician was consulted. Written informed consent was taken from the respondents. Data was entered into Microsoft office Excel $®$ software in codes and analysis was done by SPSS software version 14.0 (®. Descriptive statistical analysis, which included frequency, mean and percentages, was used to characterize the data.

${ }^{1}$ Intern Doctor, Dhaka Medical College, Bangladesh 
Approval for this study was obtained from Dhaka Medical College Ethics Committee. The author followed the recommendations of the Strengthening the Reporting of Observational Studies in Epidemiology (STROBE) Statement in writing this article.?

\section{Results}

The majority of the infants were between nine months to one year of age. The majority of the mothers (91 mothers, 42.9\%) were educated up to the secondary level and 53 mothers $(25.0 \%)$ were educated up to the primary level. The majority of the fathers $(30.7 \%)$ received education up to the secondary level, and 46 fathers $(21.7 \%)$ received primary education. The numbers of illiterate fathers and mothers were $17(8.0 \%)$ and $13(6.1 \%)$, respectively. The study revealed that $13.7 \%$ of fathers and $13.2 \%$ of mothers could only sign their names.

The majority (94.8\%) of the 212 mothers surveyed breastfed their infant (Table 1). The percentage of prelacteal feeding was $33.0 \%$. The majority of the mothers who gave prelacteal feeding used honey $(38.6 \%)$ and sugar water $(30.0 \%)$. The rate of colostrum feeding to infants was $88.7 \%$. The reason for not giving colostrum was mostly due to maternal illness (79.2\%). The rate of exclusive breast feeding was $40.6 \%$, with $97.3 \%$ of mothers starting complementary feeding when their infant reached six months of age. The most common reason for not giving breast milk was maternal illness $(72.7 \%)$. Among the 11 mothers who did not breast-fed their infants, $72.7 \%$ gave infant formula and $27.3 \%$ gave cow's milk. For giving alternate food, $45.5 \%$ mothers bottle-fed their infants, while the rest used a bowl and a spoon. In regard to the method of cleaning feeding utensils, $27.3 \%$ mothers used only water and $72.7 \%$ mother used soap and water. Before feeding the infants, $96.2 \%$ of mothers washed their hands. For those who washed their hands, 34.3\% used only water, while the rest used soap water.

\section{Discussion}

Proper feeding practices during infancy are essential, and the maintenance of these practices is encouraged worldwide due to its positive impact on child health. ${ }^{3}$ This study reveals that among the type of pre-lacteal feeding, honey (38.6\%) and sugar water $(30.0 \%)$ were the most common. A study conducted in Thailand showed that mustard oil was the most common type of prelacteal feeding $(42.9 \%)$, with honey $(23.6 \%)$, sugar water $(3.2 \%)$ and other types of food $(26.7 \%)$ being used as well. ${ }^{8}$

The percentage of mothers who gave colostrum to their in fant was $88.7 \%$ in this study. This finding differs from a study conducted in the outpatients department at a tertiary hospital in Bangladesh in 2009, which showed that $79.2 \%$ of mothers gave colostrums to their infants. ${ }^{9}$ This is an encouraging result and suggests that the awareness among the mothers regarding providing colostrum to infants is increasing. However, this difference may also be due to place and time discrepancy.

Among all infants, $94.8 \%$ were breastfed. This finding is consistent with another study conducted in rural Bangladesh, where $99.4 \%$ of the mothers breastfed their infants. ${ }^{10}$

The study reveals that $70.8 \%$ infants were breast-fed within one hour of birth. In a study published in 1996 , only $9 \%$ of mothers had initiated breast feeding immediately after birth. ${ }^{10}$ Another
Table 1. Feeding practices among infants.

\begin{tabular}{|lrr|}
\hline Categories & Freq. & $\%$ \\
\hline Pre-lacteal feeding practices $(\mathrm{n}=212)$ & 70 & 33.0 \\
\hline Type of pre-lacteal food $(\mathrm{n}=70)$ & & \\
\hline Honey & 27 & 38.6 \\
\hline Sugar water & 21 & 30.0 \\
\hline Water & 4 & 5.7 \\
\hline Others & 18 & 25.7 \\
\hline History of giving colostrum $(\mathrm{n}=212)$ & 188 & 88.7 \\
\hline Causes of not giving colostrum $(\mathrm{n}=24)$ & & \\
\hline Mother's illness & 19 & 79.2 \\
\hline Ignorance & 05 & 20.8 \\
\hline History of Breast Feeding $(\mathrm{n}=212)$ & 201 & 94.8 \\
\hline Causes of not giving breast milk $(\mathrm{n}=11)$ & & \\
\hline Mother's illness & 8 & 72.7 \\
\hline Lack of breast milk & 3 & 27.3 \\
\hline Type of food alternate to breast milk $(\mathrm{n}=11)$ & & \\
\hline Infant Formula & 8 & 72.7 \\
\hline Cow's Milk & 3 & 27.3 \\
\hline
\end{tabular}

Type of utensil used for giving food alternate to breast

milk ( $\mathrm{n}=11)$

\begin{tabular}{|ccc|}
\hline Bowl a spoon & 6 & 54.5 \\
\hline Bottle & 5 & 45.5 \\
\hline Method of cleaning the utensil $(\mathrm{n}=11)$ & & \\
\hline Soap and water & 8 & 72.7 \\
\hline Only water & 3 & 27.3 \\
\hline Time of starting breast-feeding $(\mathrm{n}=201)$ & & \\
\hline Within 1 hour & 150 & 74.6 \\
\hline Within 24 hours & 36 & 17.9 \\
\hline After 24 hours & 15 & 7.5 \\
\hline
\end{tabular}

Feeding practices of mother for their infants up to 6

months ( $n=212)$

\begin{tabular}{lrr} 
Breast milk Ct supplementary food & 115 & 54.3 \\
\hline Only breast milk (exclusively breast feeding) & 86 & 40.6 \\
\hline Only supplementary food & 11 & 5.2 \\
\hline History of starting complementary feeding after 6 mon- & 84 & 97.3
\end{tabular}

ths $(n=86)$

\begin{tabular}{lrr|}
\hline Hand washing practice before feeding the infant $(n=212)$ & 204 & 96.2 \\
\hline Hand washing technique $(n=204)$ & 134 & 65.7 \\
\hline Water and soap & 70 & 34.3 \\
\hline Only with water & 202 & 98 \\
\hline Washing of utensil before infant feeding $(n=206)$ & & \\
\hline
\end{tabular}

study conducted in a rural area of Bangladesh in 1996 showed that $73 \%$ of mothers initiated breast feeding within the first two days." But national findings in Bangladesh showed that only $43.0 \%$ newborns are put to breast right after birth. ${ }^{4}$ These encouraging results suggest that the awareness of the benefits of breast feeding among mothers has increased.

Regarding feeding practices, the majority (54.3\%) of mothers fed their infants breast milk along with supplementary food. Bengal national figures shows that only $25.0 \%$ mother exclusively breast-fed their infant by the fourth month of their li- 
ves, compared to $40.6 \%$ of mothers in the current study. Other studies reported that approximately one third of mothers exclusively breastfed their infants until two to three months of life. $3,5,12$ These results show that the prevalence of exclusive breast feeding has improved.

Furthermore, $97.3 \%$ mother started complimentary feeding after six months. However, this finding is inconsistent with another study which was conducted between 2001-2003, where $66.7 \%$ of infants were fed with complimentary food. This discrepancy may be due to time factor between the two studies. Mothers may now be more aware of the need to provide complementary food. ${ }^{3}$

For giving alternate food, $45.5 \%$ of mothers used bottle and the rest used a bowl and a spoon. In regard to the method of cleaning feeding utensils, $27.3 \%$ of the mothers used only water and $72.7 \%$ used soap and water. This finding reflects their knowledge on the importance of cleanliness in preventing diseases. It may also be due to their access to cleaning supplies.

The study findings showed that before feeding the infants, $96.2 \%$ of mothers washed their hands. For those who washed their hands, $34.3 \%$ used only water, while the other $65.7 \%$ used soap water. Used utensils were washed before feeding by $98 \%$ of mothers. A study published in 2008 showed that fewer than
$5 \%$ of caregivers wash their hands with soap before feeding babies. ${ }^{13}$ This also reflects their knowledge on the importance of hand washing. This is probably due to effective communication messages and easy availability of water and soap as showed in a study performed in Bangladesh in 2011. ${ }^{14}$

The study has several limitations. First of all recall bias may exist. Data were collected from a part of selected rural community and may not reflect the whole scenario of feeding practices in rural communities of Bangladesh, limiting the generalizability of the results. The target sample size could not be reached, and this represents another limitation. Nevertheless, this study is the first study on infant feeding practices conducted in the study area. Larger studies should be carried out to reveal the whole scenario of feeding practice in rural communities in Bangladesh and other developing countries to improve the healthcare of children.

The study demonstrates that most mothers breastfeed their infants. The percentage of giving colostrum to the infants was satisfactory. The percentages of hand washing and cleaning of utensils were also satisfactory. As the rate of exclusive breast feeding was not satisfactory, measures should be taken to promote female education and to raise awareness among the population about the importance of exclusive breast feeding during the first six months of life. 